Chirurg 2015 · 86:624-625

DOI 10.1007/s00104-015-0040-x

Online publiziert: 23. Juni 2015

(c) Springer-Verlag Berlin Heidelberg 2015

U. Settmacher

Klinik für Allgemein-, Viszeral- und Gefäßchirurgie, Universitätsklinikum Jena, Jena, Deutschland

\title{
Komplikationen nach gefäßchirurgischen Interventionen
}

\section{Der Gefäßchirurg muss das Spektrum und die Therapiemöglichkeiten beherrschen}

Invasivität kann es zu einer Verschlechterung vorbestehender Befunde kommen.

Hauptprobleme in allen anatomischen Gebieten sind insbesondere akute Ischämien nach Rekonstruktionen, die eine Nekrose der distal der Rekonstruktion gelegenen Körperregion in Abhängigkeit von der Ausprägung von Kollateralen nach sich ziehen kann. Bis heute ausgesprochen schwierig und oft vital bedrohlich ist die Infektion von Gefäßinterponaten. Die Therapie der Gefäßinfektion ist in allen Körperregionen auch heute sehr anspruchsvoll.

Zum Management von Komplikationen nach Rekonstruktionen der Aorta positionieren sich Kleinspehn et al. Hier hat heute die endovaskuläre Therapie einen hohen Stellenwert und für viele Pathologien der konventionellen Chirurgie bereits den Rang abgelaufen. Die Protheseninfektion und die intestinale Ischämie sind potenziell letale Komplikationen und oft nur mit großem Aufwand und bei früher Diagnose zu therapieren, bevor der Patient in einem septischen Multiorganversagen ist. Die Rate an spinalen Ischämien lässt sich durch entsprechende präventive und akutmedizinische Maßnahmen verringern. Als prozedurentypische Komplikationen können Endoleaks nach endovaskulärer Reparatur (EVAR) auftreten, weshalb langfristige computertomographische (CT-)Verlaufskontrollen erfolgen sollten. Die negativen Folgen einer übermäßigen Kontrastmittelapplikation bei bestehender (chronischer) Nierenfunktionseinschränkung sind bekannt.
Man nimmt an, dass dies die Hauptursache für eine langfristige Verschlechterung der Nierenfunktion bei EVAR-Patienten ist.

Zum Spektrum und zur Therapie der Komplikationen nach Rekonstruktionen an supraaortalen Gefäßen berichtet der Beitrag von T. Bürger. Hier zeigt sich, dass insbesondere für die häufigste Rekonstruktion zur Behandlung von Stenosen der Arteria carotis interna endovaskuläre Techniken laut Studienlage noch nicht den Durchbruch erzielt haben. In Deutschland werden die Daten zur Karotisrevaskularisation im Rahmen der vorgeschriebenen Qualitätssicherung beim AQUA-Institut erfasst und ausgewertet. Evidenzbasierte Unterschiede zwischen den verschiedenen Behandlungsoptionen mit den daraus resultierenden Komplikationen bestehen meist nicht. Vor- und Nachteile der Allgemein- und Regionalanästhesie sowie der Shuntverfahren zur Vermeidung neurologischer Komplikationen werden ebenfalls diskutiert. Bei einer Blutungskomplikation ist eine frühzeitige Revision angezeigt. Über $50 \%$ aller perioperativen Schlaganfälle entstehen durch eine arterielle Thrombose mit oder ohne zerebrale Embolie. Meist liegt hier ein operationstechnischer Fehler vor. Bei Verdacht auf das Bestehen einer akuten arteriellen Thrombose im Bereich der rekonstruierten Gefäßabschnitte ist die Indikation zur sofortigen Reoperation und ggf. Lyse großzügig zu stellen. Zerebrale postoperative Hyperperfusionen und Blutungen werden zunächst konservativ thera- 
piert. Zugangsbedingte lokale Komplikationen nach endovaskulären Verfahren sind Blutungen inklusive retroperitonealer Hämatome, das Aneurysma spurium, periphere Ischämien und a.v. Fisteln. Daneben stehen die verfahrensimmanenten Probleme wie die Restenosen oder auch Verschlüsse der Rekonstruktion.

\section{》) Das optimale Komplikations- management beginnt mit der primären Prophylaxe}

Zum aktuellen Stand der Behandlung von Komplikationen nach Rekonstruktionen peripherer Gefäße berichten Rückert et al., nachdem dazu letztmalig 2009 von Diener et al. in Der Chirurg und Hürlimann et al. insbesondere nach endovaskulären Rekonstruktion 2012 in der Deutschen Medizinischen Wochenschrift referiert wurde. Auch hier gilt der wichtige Grundsatz, dass das optimale Komplikationsmanagement mit der primären Prophylaxe beginnt und des Weiteren die zeitgerechte Diagnostik und Therapie von eingetretenen Komplikationen beinhaltet. Ungelöst bzw. auch durch ein optimales Blutgerinnungsregime nicht immer lösbar scheint das Vermeiden des Frühverschlusses zu sein. Auch hier ist die Infektion eine Komplikation von großer Tragweite, die meist die Explantation des alloplastischen Materials erfordert und eine alternative Revaskularisation erfordert. Nach endovaskulärer Therapie finden sich auch die zugangsbedingten Komplikationen. Hinzu kommen im Speziellen Device-spezifische Komplikationen, die aus einer technischen Fehlfunktion des verwendeten Systems resultieren.

Zum Management von Komplikationen nach Rekonstruktionen viszeraler Gefäße positionieren sich im letzten Beitrag Zanow et al. Diese werden aus unterschiedlicher Indikation isoliert oder kombiniert mit Aortenrekonstruktionen durchgeführt und stellen heute ein Gebiet dar, das offen oder endovaskulär und von Gefäß- oder Viszeralchirurgen indikationsabhängig versorgt wird. Ischämie und Infektion sind hier akute und oft lebensbedrohliche Komplikationen. Schon die Diagnostik der Komplikationen ist mitunter in der Frühphase schwierig.
Die Laparotomie ermöglicht meist neben Kontrastmittel-CT für die Leber und Pankreas oder Endoskopie für den Darm (insbesondere Innenschicht) die beste Beurteilbarkeit einer ischämischen Schädigung. Bei derzeit oft antizipierten Vorteilen der endovaskulären gegenüber der offenen Rekonstruktionen hinsichtlich des Risikos postoperativer allgemeiner wie lokaler Komplikationen und damit auch dem möglichen Vorteil in der Behandlung multimorbider Patienten relativiert sich dies hier durch die hohe Rate an Stenosen und notwendigen Revisionen im späteren Verlauf. Die Diagnostik und Therapie der Komplikationen nach Viszeralarterienrekonstruktionen sind heute interdisziplinär. Viele Komplikationen können endovaskulär therapiert werden.

Das Themenheft soll über das Spektrum der Komplikationen der aktuellen arteriellen Gefäßtherapie informieren und hier den Stellenwert des Gefäßchirurgen und seiner Möglichkeiten im Gefäßteam darstellen. Da insbesondere die endovaskuläre Therapie sich derzeit methodisch entwickelt, ist hier auch besonderes Augenmerk auf die Kenntnis und Beherrschung möglicher Komplikationen zu richten.

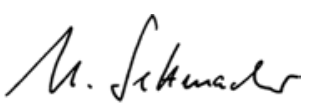

Prof. Dr. Utz Settmacher

\section{Korrespondenzadresse}

\section{Prof. Dr. U. Settmacher}

Klinik für Allgemein-, Viszeral- und

Gefäßchirurgie

Universitätsklinikum Jena

Erlanger Allee 101, 07743 Jena

utz.settmacher@med.uni-jena.de

\section{Einhaltung ethischer Richtlinien}

Interessenkonflikt. Utz Settmacher gibt an, dass kein Interessenkonflikt besteht.

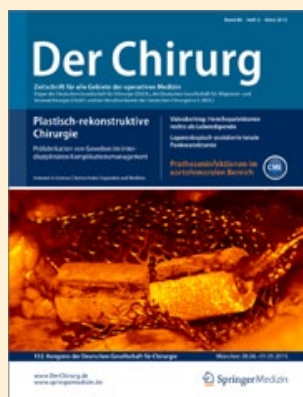

\section{4. „Julius-Springer-Preis für Chirurgie" gestiftet von SpringerMedizin}

Der 2012 ins Leben gerufene „JuliusSpringer-Preis für Chirurgie" wird auch im kommenden Jahr von Springer Medizin verliehen. Der mit 3000 EUR dotierte Preis soll die wissenschaftliche Forschung auf dem Gebiet der Chirurgie unterstützen und insbesondere den Nachwuchs fördern. Prämiert wird die beste frei eingereichte Arbeit der Rubriken „Originalien" oder "Übersichten", die im Jahr 2015 in Der Chirurg publiziert wurde. Beurteilt werden die Beiträge durch die Schriftleitung und das Herausgeberboard von Der Chirurg. Als Anerkennung und Wertschätzung der Leistungen unserer Autoren wird zudem im Rahmen eines festlichen Abends im Zuge des DGCH-Kongresses 2016 der Leserpreis für die jeweils herausragendste Arbeit der Rubriken "Leitthema" und "CME Zertifizierte Fortbildung" vergeben.

Möchten auch Sie eine „Übersicht“ oder "Originalie“" für Der Chirurg einreichen?

Um Ihnen bei der Manuskripterstellung behilflich zu sein, haben wir für unsere Autoren ausführliche Autorenleitfäden und Musterbeiträge zusammengestellt. Diese und weitere Hinweise zur Manuskripterstellung finden Sie auf www.DerChirurg.de

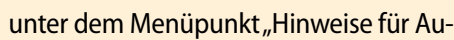
toren".

Bitte reichen Sie Ihren fertigen Beitrag online über unser Begutachtungssystem Editorial Manager ein: http://dech.edmgr.com/

Wir freuen uns auf Ihre Beiträge! Ihre Redaktion Der Chirurg 